\title{
Promoting awareness of depression with a mobile application: a usability study and evaluation
}

\begin{abstract}
This paper reports selected results from a study designed to understand the potential and limitations of a mobile application in promoting health awareness of depression to patients, their families and others. The mobile application was developed with a user-centered approach, which emphasized user preferences and opinions throughout an iterative process of development. It comprised medical information about depression, and causes, symptoms and methods of treatment. We conducted several design and evaluation sessions with a group of users (patients, their family, doctors, nurses and general users). Our findings demonstrate that the mobile medical application has the potential to improve and increase awareness among patients and their family members.
\end{abstract}

Keyword: Depression; Health awareness; Mobile medical apps 